\title{
ES Research Square

\section{Bioinformatics analysis of potential common molecular pathogenesis assumed by intracranial aneurysm, aortic aneurysm and aortic dissection}

Chao Zhao ( $D$ 32279950@qq.com )

Rizhao People's Hospital https://orcid.org/0000-0003-3552-7737

Xinchun Cui

Qufu Normal University - Rizhao Campus

Guodong Liu

The Second Affiliated Hospital of Chongqing Medical University

Jianlong Li

Rizhao People's Hospital

Jinxing Liu

Qufu Normal University - Rizhao Campus

Junliang Shang

Qufu Normal University - Rizhao Campus

Shuai Wang

Rizhao People's Hospital

Ronghua Shi

Rizhao People's Hospital

Aihong Wu

Qufu Normal University

\section{Research Article}

Keywords: Intracranial aneurysm, Abdominal aortic aneurysms, Aortic dissection, Bioinformatics, Differentially expressed genes, Protein-protein Interaction network

Posted Date: April 22nd, 2021

DOI: https://doi.org/10.21203/rs.3.rs-417952/v1

License: (1) (1) This work is licensed under a Creative Commons Attribution 4.0 International License.

Read Full License 


\section{Abstract}

This study is intended to find possible pathogenesis-related genetic overlap and common molecular mechanisms of intracranial aneurysm, abdominal aortic aneurysm and aortic dissection. Three mRNA microarray datasets,GSE75436 of intracranial aneurysms, GSE7084 of abdominal aortic aneurysm and GSE52093 of aortic dissection were downloaded from Gene Expression Omnibus and detected in silico . DEGs of these three datasets screened through GEO2R, respectively. The overlapping genes were found by Venny mapping. Subsequently, Gene Ontology, Kyoto Encyclopedia of Genes and Genomespathway enrichment analysiswere performed using the DAVID database and protein-protein interaction network analyses were conducted by STRING and Cytoscape webpage tool to illustrate the molecular mechanisms in their pathogenesis and progression.This study identified 178 DEGs, including SMTN, MYH11, TAGLN, ACTG2, CNN1, MYLK, LMOD1, MYL9,VCL and ACTC1 in the the most significant module. Except for those confirmed biological processes, mesenchyme migration and platelet aggregation are common biological processes shared by genes in the most significant module and the hub genes. Focal adhesionssignaling pathway is highlighted in this analysis. The present study identified possible pathogenesis-related genetic overlap and common molecular mechanisms of intracranial aneurysm, abdominal aortic aneurysm and aortic dissection, which may contribute to their diagnosis, treatment and prognostic prediction with a systematic view.

\section{Introduction}

Intracranial aneurysm (IA), abdominal aortic aneurysms (AAA) and aortic dissection (AD) have yet lingered as life-threatening diseases. Currently, endovascular intervention and surgical treatment are the two main preventative methods for them. The molecular mechanisms leading to their initiation, progression and rupture remain incompletely interpreted and were discussed respectively in the past. As a result, no safe and effective noninvasive IA, AAA or AD therapies have been identified and implemented in clinical practice by now.AD and thoracic aortic aneurysm(TAA) share common etiologies and feature common pathological characteristics to a great extent ${ }^{1}$.Generally IAs have saccular shapes, whereas AAAs and TAAs are more often spindle-shaped; Atherosclerosis plays a more significant role in AAA, compared with IA and TAA ${ }^{2,3}$.Although these three diseases have different internal molecular mechanisms leading to different clinical manifestations of occurrence and development ${ }^{4,5}$, they are also related in genetic and pathological processes. For example, the prevalence of IA is higher in patients with $A D$ or aortic aneurysm; Most aneurysms are named according to the local parent vessels, but they are often accompanied with systematic vascular lesions ${ }^{6}$.

Expression microarray technology and bioinformatic analysis for genetic dysfunction research have been extensively applied so far, identifying the differentially expressed genes (DEGs) and signal pathways leading to the pathogenesis and progression of many diseases. In order to understand the common molecular mechanisms among these three diseases better, the author research them via bioinformatics methods. In this study, three mRNA microarray datasetsfrom Gene Expression Omnibus (GEO) were 
included and detected to reveal common DEGs among IA, AAA and AD. Subsequently, Gene Ontology(GO), Kyoto Encyclopedia of Genes and Genomes (KEGG) pathway enrichment analysis and protein-protein interaction(PPI) network analyses were conducted to illustrate the molecular mechanisms in their pathogenesis and progression.

\section{Materials And Methods}

\section{gene expression datasets}

Three gene expression profiles datasets, GSE75436, GSE7084 and GSE52093, were identified following key word search of "aneurysm", "expression profile by array" and "human"in the Gene Expression Omnibus (GEO) (https://www.ncbi.nlm.nih.gov/geo/query/acc.cgi) ${ }^{7}$ and downloaded. Gene expression profile dataset GSE75436 contains 15 IAs wall tissues samples and their matched superficial temporal artery wall tissues samples, based on platform GPL570 (Affymetrix Human Genome U133 Plus 2.0 Array). Gene expression profile dataset GSE7084 includes 8 abdominal aortic aneurysm samples and 7 non-diseased abdominal aortas control samples, based on platform GPL2507(Illumina Sentrix Human-6 Expression BeadChip).Gene expression profile dataset GSE52093 is composed of 7 dissected ascending aorta samples and 5 normal ascending aorta control samples, based on platform GPL10558 (Illumina HumanHT-12 V4.0 Expression BeadChip).

\section{Identifying differentially expressed genes}

Gene expression data tables of GSE75436ロGSE7084 and GSE52093 were obtained via GEO2R webpage application (http://www.ncbi.nlm.nih.gov/geo/geo2r/), which is an R programming languages-based tool for identifying $\mathrm{DEGs}^{7}$. In GEO2R, query for each datasets were conducted with defining and assigning "disease" and "control" groups and selecting "Top 250" DEGs, respectively. Difference of $p$ value 00.05 and $\mid \log _{2} \otimes f$ foldchange $\mathbb{\|} \mid 1$ is considered statistically significant. Common differently expressed genes 囚cDEGs\of these three datasets, the overlapped genes, are illustrated via Venny mapping (https://bioinfogp.cnb.csic.es/tools/venny/).

\section{Functional and pathway enrichment analysis}

The Database for Annotation,Visualization and Integrated Discovery Version 6.8 (DAVID) was used to perform GO term and KEGG pathway analysis for the cDEGs (https://david.ncifcrf.gov/).Gene Ontology (GO) enrichment annotation is to locate functional gene products at the subcellular structures(cellular component $₫ \mathrm{CC}$ ), describe activities that occur at the molecular level(molecular function, MF) and the larger processes accomplished by multiple molecular activities (biological process $₫ \mathrm{BP}$ ). The Kyoto Encyclopedia of Genes and Genomes (KEGG) pathway database, analyzing the potential relevant biological function, provides useful structured information of a gene network. $p$ value $<0.05$ and FDR $<0.05$ was the cut-off criteria in GO and KEGG pathway analysis. 
The PPI networks and analysis of most significant moduleThe Search Tool for the Retrieval of Interacting Genes (STRING) database Version 11.0 (http://string-db.org/) is a database of known and predicted PPI, consisting of direct (physical) associations and indirect (functional) associations ${ }^{8}$. Input the gene symbols of cDEGs into the "multiple proteins" selection bar of STRING『and then the interactions between proteins encoded by cDEGs acquired with cut-off criterion "combined score $>0.4$ ". Then Cytoscape Version 3.7. $2^{9}$ were utilized to visualize the PPI network of the cDEGs. Molecular Complex Detection (MCODE) plugin was used to select the most significant module from the PPI network with degree cutoff $=2$, haircut on, node score cutoff $=0.2, \mathrm{k}$-score $=2$, maximum depth $=100$, and nodes more than 5 . The GO and KEGG pathway enrichment analyses for genes in this module were performed using DAVID.Hub genes selection and analysis.CytoHubba is a plugin software of Cytoscape. It predicts and explores important nodes and sub-networks in a given network by 12 topological analysis methods including Degree, EPC, MNC, et al. The hub genes, acting more importantly in biological processes than the other DEGs, were selected with degrees $\geq 10$ by CytoHubba. Then,the biological process analysis of them was performed and visualized using Biological Networks Gene Ontology tool (BinGO) plugin of Cytoscape with the significance level 0.02 . BiNGO is a software to select statistically overrepresented Gene Ontology (GO) categories in a set of genes or a subgraph of a biological network, and maps the predominant functional themes of a given gene set on the GO hierarchy. GO and KEGG function analysis of these hub genes were performed in DAVID ( $p$ value $<0.05$ ).

\section{Results}

\section{Identification of DEGs}

A total of 1963, 515 and 853 DEGs were extracted from GSE75436, GSE7084 and GSE52093, respectively

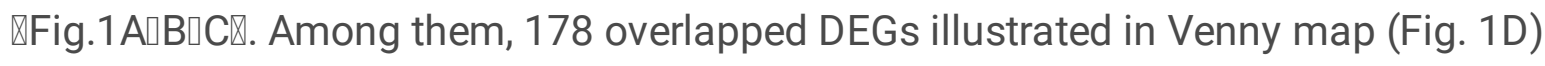

\section{Functional and pathway enrichment analysis of DEGs}

The functional annotation of CDEGs was conducted via DAVID in order to understand the subcellular localization, molecular functions, biological processesof cDEGs. Functional enrichment analysis showed that CDEGs were enriched in cellular components(CC) such as $Z$ disc, focal adhesion, extracellular space, et al; For GO molecular functions(MF), the cDEGs were significantly enriched in actin binding, structual constituent of muscle, actin filament binding, protein kinase activity, et al; Upon biology processes(BP), the CDEGs were significantly enriched in muscle contraction, chemotaxis, cell chemotaxis, cell adhesion, et al.Furthermore, KEGG analysis revealed that CDEGs were significantly enriched in vascular smooth muscle contraction, neurotrophin singnaling pathway, cGMP-PKG signaling pathway, osteoclast differentiation, et al.( Top 10 in Fig.2 A, B, C; Top 13 in Fig.2 D; Both according to -log10(p-value)).

\section{PPI network construction and module analysis}

The PPI network of cDEGs was mapped intuitively and expansively to illustrate the possible association between down-stream proteins with 137 nodes and 336 edges (Figure 3A). After MCODE plugin of 
Cytoscape processing, the most significant module was selected with nodes $=10$ and edges $=43$ (Fig. 3B),

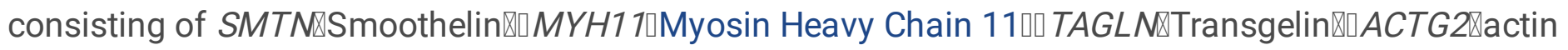

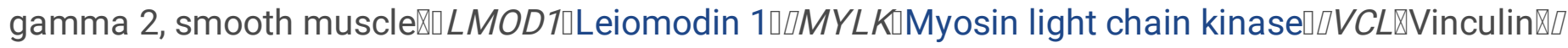

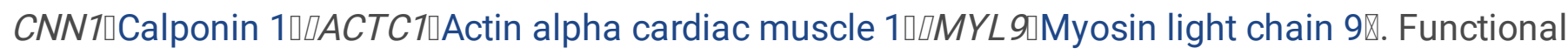
enrichment analysis indicated that these genes in the module were enriched in GO terms and KEGG pathways in Table 1

\section{Functional and pathway enrichment analysis of hub genes}

A total of 20 hub genes were screened out, and their gene symbols and aliases were shown in Table2 with PPI network in Fig.4A.The biological processes resulted from BingGO analysis with different color depths of each node according to their $p$ value (Fig.4B). The functional analysis of hub genes was conducted through DAVID (Table 3). Muscle contraction, smooth muscle contraction, actomyosin structure organization, inflammatory response co-existed in Fig.4B and Table 3.

\section{Discussion}

Thoracic aortic diseases (TADs) include thoracic aortic aneurysms (TAAs) and aortic dissections (ADs), associating with a widely common genetic etiology, and are usually discussed as a whole by many scholars ${ }^{10,11}$. TADs are different from abdominal aortic aneurysms (AAAs) in clinical features, inheritance modes, et al. But TADs and AAAs share several joint pathogenic processes, for example, proteolytic elastic tissue degeneration and smooth muscle dysfunction ${ }^{5}$. In an autopsy study, these thoracoabdominal artery lesions can co-exist ${ }^{12}$. So, there may be same pathogenic molecular mechanisms between TADs and AAAs.The prevalence of IA in patients with aortic dissection or aneurysms is higher than those without ${ }^{13,14,15}$.Degeneration of the elastic tissueand smooth muscle dysfunction are also explicated in IAs formation and rupture ${ }^{16}$. These evidences suggest associations in these diseases pathogenetic process, but still understood unclearly. In this paper, the common differentially expressed genes of the three diseases IA, AD and AAA, and their possible signaling pathways was detected in silico, so as to provide basis for future studies.

In this study, to reveal possible common pathogenesis-related genes of IA, AAA and AD, the author selected three microarray profile datasets ,GSE75436 of IAs, GSE7084 of AAAs and GSE52093 of ADs. DEGs of these three datasets screened through GEO2R, respectively. In summary, 178 common DEGs of the three datasets were identified. The enrichment analysis demonstrated these DEGs were primarily enriched in biological processes, including muscle cell contraction, cell chemotaxis, cell adhesion and protein localization to cell surface.To date, smooth musclecells (SMCs), leukocytes, complements, immunoglobulins, cytokines have been affirmed to be contributors to IA pathogenesis. Their roles in the biological processes mentioned above need further investigation. In $\mathrm{GO}$ cellular component enrichment analysis, the most enriched component was $Z$ disc, whereas in $\mathrm{GO}$ molecular function analysis, it was actin binding. In KEGG pathway analysis, DEGs were mostly enriched in vascular smooth muscle contraction, neurotrophin signaling pathway, cGMP-PKGsignaling pathway, osteoclast differentiation, 
arginine and proline metabolism, et al. in Fig.2D. Only smooth muscle contraction, Toll-like receptor signaling pathway and CGMP-PKG signaling pathway have been researched extensively by now ${ }^{17,18,19}$.

SMCs contractile dysfuction (dedifferentiation) refers to the degeneration from an initial contractile status to a inflammatory and matrix remodeling status under the stimulation of the cellular and extracellular environment. It is an integral part of the inflammatory response along with subsequent cell death. It is a key predisposing process of aortic dissection, aortic aneurysms and intracranial aneurysms $20,21,22,23$. Genes in the most significant module of Fig. 3B are all embedded in hub genes of Fig. 4A, suggesting that their biological function is particularly important. They are all associated with SMC contraction, but only MYH11 and MYLK have been validated to be predisposing genes of aneurysmal diseases and $A D$ further ${ }^{5,24}$.

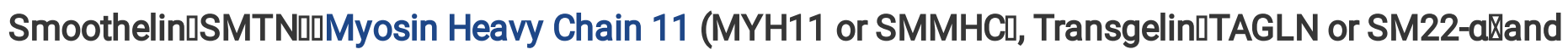
CNN1 are SMC differentiation biomarkers that function importantly in SMC contraction. SMTN family are composed of SMTN-a, SMTN-band SMTNL1.SMTN was expressed in tunica media SMCs of normal arteries , but unexpressed in the media SMCs of saccular intracranial aneurysm (sIA); Meanwhile, sIA SMCs showed significant decrease of $M Y H 11$ expression ${ }^{25}$. It's reported that $M Y H 11$ mutation correlated with thoracic aortic aneurysms, dissections and de novo intracranial aneurysm formation ${ }^{26}$.TAGLN is an actin binding enzyme of the calponin family, performing actin binding and actin bundling activities. Loss of TAGLN promotes thoracic aortic aneurysm and aortic dissection progression in vivo ${ }^{27}$. But no association with its roles and other vascular diseases has been reported yet.

in vitro experiments implicated that $A C T G 2$, a target of miR-193a-3p, participated in the development of $A D^{28}$. Feng $\mathrm{J}$ reported $C N N 1$ was downregulated in aortic dissection vascular smooth muscle cells $\triangle$ which might be a key pathogenetic gene in vascular diseases ${ }^{29}$. LMOD1 is a highly specific contractile gene for smooth muscle lineages. myosin light chain 90MYL9Dis involved in inflammatory response, which is a main biological process of aneurysmal diseases ${ }^{30}$. Defects of cytoskeleton proteins VCL and actin ACTC1 are the cause of dilated cardiomyopathy. These genes in the most significant module except MYH11 and MYLK have not been discussed in aneurysmal diseases and need continous research in the future.

In this study, smooth muscle contraction, actomyosin structure organization, mesenchyme migration and platelet aggregation are common biological processes shared by genes in the most significant module and hub genes. Smooth muscle contraction, actomyosin structure organization are important in preventing aneurysm (or dissections) pathogenesis, validated by many researches ${ }^{5}$. Our findings are in accordance with previously reported data on the role of vascular SMCs in aneurysm pathogenesis. Nevertheless, mesenchyme migration and platelet aggregation have not been understood completely. Platelet aggregation and thrombosis are the subsequent events of elastin integrity loss after AAA initiation. Expounded by quite limited articles, platelet aggregation plays a significant role in AAA onset and progression, and platelet inhibitors can attenuate aneurysm formation in rat AAA model, though 
lacking of clinical evidence ${ }^{31}$. Inflammatory factors TGF $\beta$ released during platelet aggregation, can promote the transformation of SMC phenotype from contractile status to inflammatory status, along with inflammatory responses, thereby contribute to aneurysm formation ${ }^{32}$. These evidences lead us to speculateon a positive feedback between aneurysm pathogenesis and platelet aggregation.

Focal adhesion signaling pathway co-exhibited in Fig 2D, Table 10Table 3. Focal adhesions function as the transmembrane signal transduction interface between extracellular matrix and intracellular cytoskeleton, and has not been elucidated extensively in aneurysm pathogenesis. Testin (TES), a focal adhesion scaffold protein, was validated as a potential contributor to TAA onset ${ }^{33}$. THSD1, a novel nascent adhesion protein, is provided likely to cause intracranial aneurysm in both familial and sporadic patients $^{34}$. MYL9, VCL and MYLK mined in this article may play significant roles in aneurysmal diseases pathology via focal adhesion pathway, which worthy of further investigation.

Genetic variation is animportant factor of vascular diseases, and the understanding of its role is gradually enriched. It can lead to biological degeneration in blood vessels, including cellular and tissue events contributing to intracranial and aortic aneurysms (or dissection), which may provide new therapeutic targets. Genetic testing is regular procedure of clinical diagnosis and treatment. The genes discussed in this paper, may be developed as potential predictors of systemicaneurysms in genetic test or targets of vascular therapy.

There are some limitations to this study. The first one is merely single-aspect analysis. Other methods, e.g. gene set enrichment analysis (GSEA), can provide understanding of genes function from another aspect. The second one is lacking experimental and clinical feature validation, such as in vitro and in vivo experiments, patients' age, sex, different types of aneurysms, and so on. And in addition, the sample count of each group is limited. False-positive incidences in independent microarray analysis hinder reliable data acquisition.

To date, genetic sharing among IA, AAA, and AD has been considered mainly within families ${ }^{35,36}$, but possible genetic corelations in sporadic aneurysmal cases are also worthy of attention. Therefore, further research focusing on the aforementioned hub genes and biological processes calls for more evidences with a systematic view.

\section{Conclusion}

This article aiming to find possible pathogenesis-related genetic overlap of IA, AAA and AD, identified 178 DEGs, which include SMTN, MYH11, TAGLN, ACTG2, CNN1, MYLK, LMOD1, MYL9,VCL and ACTC1 in the the most significant module. Except for those confirmed biological processes, mesenchyme migration and platelet aggregation are common biological processes shared by genes in the most significant module and the hub genes. Focal adhesionssignaling pathway highlighted in this analysis, has not been elucidated extensively in aneurysm pathogenesis by now. Basic and clinical experiments are needed to 
verify all of these results that may contribute to the diagnosis, treatment and prognostic prediction of IA, $A A A$ and $A D$ with a systematic view.

\section{Declarations}

\section{Compliance with ethical standards}

\section{Conflicts of interest}

The authors declare no conflict of interest related to the content of this article

Funding: Research Support Fund for Teachers of Jining Medical University (JYFC2018FKJ144)

Conflicts of interest: The authors declare no conflict of interest related to the content of this article

Availability of data and material: Open

Code availability: Not applicable

Authors' contributions: Chao Zhao conceived and designed the study. Xinchun Cui , Guodong Liu, Jianlong Li, Jinxing Liu and Junliang Shang performed data processing. Chao Zhao, Shuai Wang and Ronghua Shi wrote the paper. Chao Zhao and Aihong Wu reviewed and edited the manuscript. All authors read and approved the manuscript.

Ethics approval: Not applicable

Consent to participate: Not applicable

Consent for publication: Not applicable

\section{References}

1.Boileau A, Lindsay ME, Michel JB, Devaux Y. $\$ 2018 \rrbracket$ Epigenetics in Ascending Thoracic Aortic Aneurysm and Dissection. Aorta (Stamford) 6(1):1-12. https://doi.org/10.1055/s-0038-1639610

2.Penn DL, Witte SR, Komotar RJ, Sander Connolly E Jr (2014) The role of vascular remodeling and inflammation in the pathogenesis of intracranial aneurysms.J Clin Neurosci 21(1):28-32. https://doi.org/10.1016/j.jocn.2013.07.004

3.Norman PE, Powell JT (2010) Site specificity of aneurysmal disease. Circulation 121(4):560-568. https://doi.org/ 10.1161/CIRCULATIONAHA.109.880724

4.Humphrey JD, Taylor CA(2008)Intracranial and abdominal aortic aneurysms: similarities, differences, and need for a new class of computational models. Annu Rev Biomed Eng 10:221-246. https://doi.org/10.1146/annurev.bioeng.10.061807.160439 
5.Pinard A, Jones GT, Milewicz DM (2019) Genetics of thoracic and abdominal aortic diseases. Circ Res 124(4):588-606. https://doi.org/10.1161/CIRCRESAHA.118.312436

6.Song J, Lim YC, Ko I, Kim JY, Kim DK(2020)Prevalence of Intracranial Aneurysms in Patients With Systemic Vessel Aneurysms: A Nationwide Cohort Study. Stroke 51(1):115-120.

https://doi.org/10.1161/STROKEAHA.119.027285

7.Barrett T, Wilhite SE, Ledoux P, Evangelista C, Kim IF, Tomashevsky M, Marshall KA, Phillippy KH, Sherman PM, Holko Met al (2013) NCBI GEO: archive for functional genomics datasets-update. Nucleic Acids Res 41(D1):D991-D995. https://doi.org/10.1093/nar/gks1193

8.Szklarczyk D, Morris JH, Cook H, Kuhn M, Wyder S, Simonovic M, Santos A, Doncheva NT, Roth A, Bork P, Jensen LJ, von Mering C (2017) The STRING database in 2017: quality-controlled protein-protein association networks, made broadly accessible. Nucleic Acids Res 45(D1):D362-D368.

https://doi.org/10.1093/nar/gkw937

9.Shannon P, Markiel A, Ozier O, Baliga NS, Wang JT, Ramage D, Amin N, Schwikowski B, Ideker T (2003) Cytoscape: a software environment for integrated models of biomolecular interaction networks. Genome Res 13(11):2498-2504. https://doi.org/10.1101/gr.1239303

10.Goldfinger JZ, Halperin JL, Marin ML, Stewart AS, Eagle KA, Fuster V (2014) Thoracic aortic aneurysm and dissection. J Am Coll Cardiol 64(16):1725-1739. https://doi.org/10.1016/j.jacc.2014.08.025

11.Isselbacher EM, Lino Cardenas CL, Lindsay ME (2016) Hereditary influence in thoracic aortic aneurysm and dissection. Circulation 133(24):2516-2528. https://doi.org/10.1161/CIRCULATIONAHA.116.009762

12.Svensjö S, Bengtsson H, Bergqvist D (1996) Thoracic and thoracoabdominal aortic aneurysm and dissection: an investigation based on autopsy. Br J Surg 83(1):68-71.

https://doi.org/10.1002/bjs.1800830122

13.Lee D, Ahn SJ, Cho ES, Kim YB, Song SW, Jung WS, et al (2017) High prevalence of intracranial aneurysms in patients with aortic dissection oraneurysm: feasibility of extended aorta CT angiography with involvement of intracranial arteries. J Neurointerv Surg 9(10):1017-1021.

https://doi.org/10.1136/neurintsurg-2016-012619

14.Jung WS, Kim JH, Ahn SJ, Song SW, Kim BM, Seo KD, et al (2017) Prevalence of intracranial aneurysms in patients with aortic dissection. AJNR Am J Neuroradiol 38(11):2089-2093. https://doi.org/10.3174/ajnr.A5359

15.Rouchaud A, Brandt MD, Rydberg AM, Kadirvel R, FlemmingK,Kallmes DF, et al (2016) Prevalence of intracranial aneurysms in patients with aortic aneurysms. AJNR Am J Neuroradiol 37(9):1664-1668. https://doi.org/10.3174/ajnr.A4827 
16.Chalouhi N, Hoh BL, Hasan D (2013) Review of cerebral aneurysm formation, growth, and rupture. Stroke 44(12):3613-3622. https://doi.org/10.1161/STROKEAHA.113.002390

17.Wang W, Li H, Yu L, Zhao Z, Wang H, Zhang D, Zhang Y, Lan Q, Wang J, Zhao J (2017) Aberrant expression of IncRNAs and mRNAs in patients with intracranial aneurysm. Oncotarget 8(2):2477-2484. https://doi.org/10.18632/oncotarget.13908

18.Kleinloog R, Verweij BH, van der Vlies $\mathrm{P}$ (2016) RNA sequencing analysis of intracranial aneurysm walls reveals involvement of lysosomes and immunoglobulins in rupture. Stroke 47(5):1286-1293. https://doi.org/10.1161/STROKEAHA.116.012541

19.Kurki MI, Hakkinen SK, Frosen J, et al (2011) Upregulated signaling pathways in ruptured human saccular intracranial aneurysm wall: an emerging regulative role of Toll-like receptor signaling and nuclear factor-KB, hypoxia-inducible factor-1A, and ETS transcription factors. Neurosurgery 68(6):16671675. https://doi.org/10.1227/NEU.0b013e318210f001

20.Wang L, Zhang J, Fu W, Guo D, Jiang J, Wang Y (2012) Association of smooth muscle cell phenotypes with extracellular matrix disorders in thoracic aortic dissection. J Vasc Surg 56(6):1698-1709, $1709 . e 1$. https://doi.org/10.1016/j.jvs.2012.05.084.

21.Ailawadi G, Moehle CW, Pei $\mathrm{H}$, et al (2009) Smooth muscle phenotypic modulation is an early event in aortic aneurysms. J Thorac Cardiovasc Surg 138(6):1392-1399.

https://doi.org/10.1016/j.jtcvs.2009.07.075

22.Inamoto S, Kwartler CS, Lafont AL, et al (2010) TGFBR2 mutations alter smooth muscle cell phenotype and predispose to thoracic aortic aneurysms and dissections.Cardiovasc Res 88(3):520-529. https://doi.org/10.1093/cvr/cvq230

23.Oka M, Shimo S, Ohno N, Imai H, Abekura Y, Koseki H, Miyata H, Shimizu K, Kushamae M, Ono I, Nozaki K, Kawashima A, Kawamata T, Aoki T (2020) Dedifferentiation of smooth muscle cells in intracranial aneurysms and its potential contribution to the pathogenesis. Sci Rep 10(1):8330. https://doi.org/10.1038/s41598-020-65361-x

24.Cheng Q, Li Z, Wang R, Zhang H, Cao H, Chen F, Li H, Xia Z, Feng S, Zhang H, Rui Y, Fan F ( 2019) Genetic profiles related to pathogenesis in sporadic intracranial aneurysm patients. World Neurosurg 131:e23-e31. https://doi.org/10.1016/j.wneu.2019.06.110

25.Matteo Coen, Karim Burkhardt, Philippe Bijlenga, et al (2013) Smooth muscle cells of human intracranial aneurysms assume phenotypic featuressimilar to those of the atherosclerotic plaque. Cardiovasc Pathol 22(5):339-344. https://doi.org/10.1016/j.carpath.2013.01.083

26.Ravindra VM, Karsy M, Schmidt RH, Taussky P, Park MS, Bollo RJ (2016) Rapid de novo aneurysm formation after clipping of a ruptured middle cerebral artery aneurysm in an infant with an MYH11 
mutation. J Neurosurg Pediatr 18(4):463-470. https://doi.org/10.3171/2016.5.PEDS16115

27.Lino Cardenas CL, Kessinger CW, MacDonald C, Jassar AS, Isselbacher EM, Jaffer FA, Lindsay ME (2018) Inhibition of the methyltranferase EZH2 improves aortic performance in experimental thoracic aortic aneurysm. JCl Insight 3(5):e97493. https://doi.org/10.1172/jci.insight.97493

28.Wang W, Wang Y, Piao H, Li B, Zhu Z, Li D, Wang T, Liu K (2021) Bioinformatics analysis reveals microRNA-193a-3p regulates ACTG2 to control phenotype switch in human vascular smooth muscle cells. Front Genet. 11:572707. https://doi.org/ 10.3389/fgene.2020.572707

29.Feng J, Ge S, Zhang L, Che H, Liang C (2016) Aortic dissection is associated with reduced polycystin-1 expression, an abnormality that leads to increased ERK phosphorylation in vascular smooth muscle cells. Eur J Histochem. 60(4):2711. https://doi.org/10.4081/ejh.2016.2711

30.Kimura MY, Koyama-Nasu R, Yagi R, Nakayama T (2019) A new therapeutic target: the CD69-Myl9 system in immune responses. Semin Immunopathol 41(3):349-358. https://doi.org/10.1007/s00281-01900734-7

31.Cameron SJ, Russell HM, Owens AP $3^{\text {rd }}$ (2018)Antithrombotic therapy in abdominal aortic aneurysm: beneficial or detrimental? Blood 132(25):2619-2628. https://doi.org/10.1182/blood-2017-08-743237

32.Starke RM, Chalouhi N, Ding D, Raper DM, Mckisic MS, Owens GK, Hasan DM, Medel R, Dumont AS (2014) Vascular smooth muscle cells in cerebral aneurysm pathogenesis. Transl Stroke Res 5(3):338-346. https://doi.org/10.1007/s12975-013-0290-1

33.Li Y, Gao S, Han Y, Song L, Kong Y, Jiao Y, Huang S, Du J, Li Y( 2021) Variants of Focal Adhesion Scaffold Genes Cause Thoracic Aortic Aneurysm. Circ Res 128(1):8-23.

https://doi.org/10.1161/CIRCRESAHA.120.317361

34.Rui YN, Xu Z, Fang X, Menezes MR, Balzeau J, Niu A, Hagan JP, Kim DH ( 2017) The Intracranial Aneurysm Gene THSD1 Connects Endosome Dynamics to Nascent Focal Adhesion Assembly. Cell PhysiolBiochem 43(6):2200-2211. https://doi.org/10.1159/000484298

35.Regalado E, Medrek S, Tran-Fadulu V, Guo DC, Pannu H, Golabbakhsh H, SmartS, Chen JH, Shete S, Kim DH, Stern R, Braverman AC, Milewicz DM (2011)Autosomal dominant inheritance of a predisposition to thoracic aortic aneurysms and dissections and intracranial saccular aneurysms. Am J Med Genet A 155A(9):2125-2130. https://doi.org/ 10.1002/ajmg. a.34050

36. Kim DH, Van Ginhoven G, Milewicz DM ( 2005) Familial aggregation of both aortic andcerebral aneurysms: evidence for a common genetic basis in a subset offamilies. Neurosurgery 56:655-661; discussion 655-661. https://doi.org/10.1227/01.neu. 0000156787.55281.53 


\section{Tables}

Table 1 GO terms and KEGG pathways analysis of genes in the most significant module

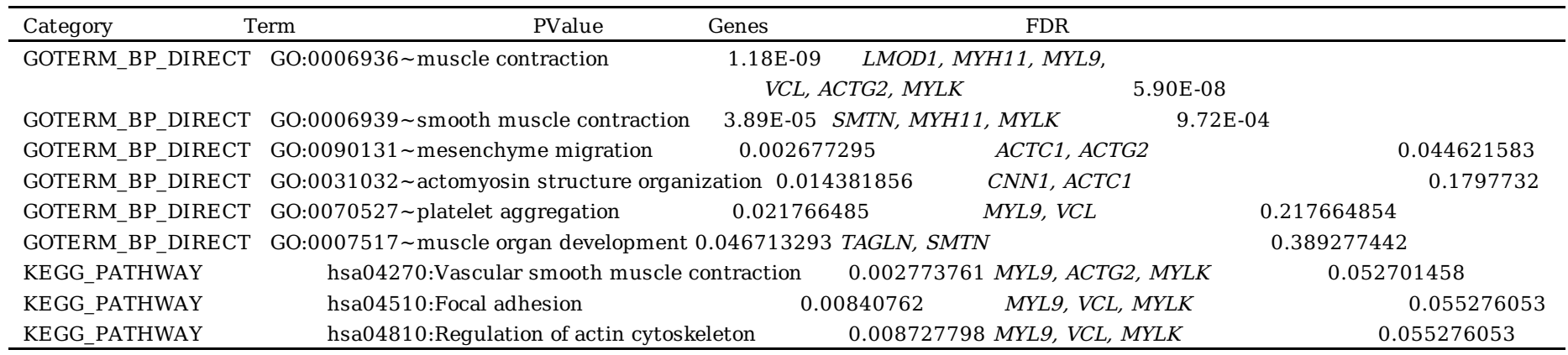

Table 2 information of hub genes

\begin{tabular}{|c|c|c|}
\hline \multicolumn{3}{|c|}{ Gene symbol $\quad$ Full name } \\
\hline$V C L$ & $C M D 1 W, C M$ & MH15, HEL114, MV, MVCL \\
\hline CXCL8 & C-X-C motif chemokine ligand 8 & $\begin{array}{l}\text { GCP-1, GCP1, IL8, LECT, LUCT, LYNAP, MDNCF, MONAP, } \\
N A F, N A P-1, N A P 1\end{array}$ \\
\hline$S P P 1$ & secreted phosphoprotein 1 & $B N S P, B S P I, E T A-1, O P N$ \\
\hline MYH11 & myosin heavy chain $11 \quad A A$ & AT4, FAA4, SMHC, SMMHC \\
\hline$T A G L N$ & Transgelin $\quad S M 22, S \Lambda$ & $\begin{array}{l}\text { M22-alpha, SMCC, TAGLN1, } \\
\text { WS3-10 }\end{array}$ \\
\hline$A C T C 1$ & actin alpha cardiac muscle 1 & $A C T C, A S D 5, C M D 1 R, C M H 11, L V N C 4$ \\
\hline FLNA & filamin A $\quad A B P-280$, & $\begin{array}{l}A B P X, C S B S, C V D 1, F G S 2, \\
F L N, F M D, M N S, N H B P, O P D, X L V D\end{array}$ \\
\hline $\begin{array}{l}\text { SPI1 } \\
\text { MYL9 }\end{array}$ & $\begin{array}{l}\text { Spi-1 proto-oncogene } \\
\text { myosin light chain } 9\end{array}$ & $\begin{array}{l}\text { PU.1, SFPI1, SPI-1, SPI-A } \\
M L C-2 C, M L C 2, M R L C 1, M Y R L 2\end{array}$ \\
\hline$M Y L K$ & myosin light chain kinase & $\begin{array}{l}\text { AAT7, KRP, MLCK, MLCK1, MLCK210, MMIHS, MSTP083, } \\
\text { SmMLCK }\end{array}$ \\
\hline ITGAX & integrin subunit alpha $\mathrm{X} \quad C l$ & $D 11 C, S L E B 6$ \\
\hline$A C T G 2$ & actin gamma 2 , smooth muscle & ACT, ACTA3, ACTE, ACTL3, ACTSG, VSCM \\
\hline$L M O D 1$ & leiomodin $11 D, 64 k D$, & $D 1, S M-L M O D, S M L M O D$ \\
\hline MYH10 & myosin heavy chain $10 \quad N$ & IMMHC-IIB, NMMHCB \\
\hline$L I L R B 2$ & $\begin{array}{l}\text { leukocyte immunoglobulin like } \\
\text { receptor B2 } \\
\text { MIR1 }\end{array}$ & CD85D, ILT-4, ILT4, LIR-2, LIR2, MIR-10, \\
\hline CXCL 1 & C-X-C motif chemokine ligand 1 & $\begin{array}{l}\text { FSP, GRO1, GROa, MGSA, MGSA-a, NAP-3, } \\
\text { SCYB1 }\end{array}$ \\
\hline$S M T N$ & smoothelin none & \\
\hline OLR1 & $\begin{array}{l}\text { oxidized low density lipoprotein } \\
\text { receptor } 1\end{array}$ & $C L E C 8 A, L O X 1, L O X I N, S C A R E 1, S L O X 1$ \\
\hline CCL5 & C-C motif chemokine ligand $5 \quad D$ & $\begin{array}{l}17 S 136 E, R A N T E S, \text { SCYA5, SIS-delta, } \\
\text { SISd, TCP228, eoCP }\end{array}$ \\
\hline CNN1 & calponin 1 & SMCC, Sm-Calp \\
\hline
\end{tabular}

Table 3 GO-BP terms and KEGG pathways analysis of hub genes by DAVID 


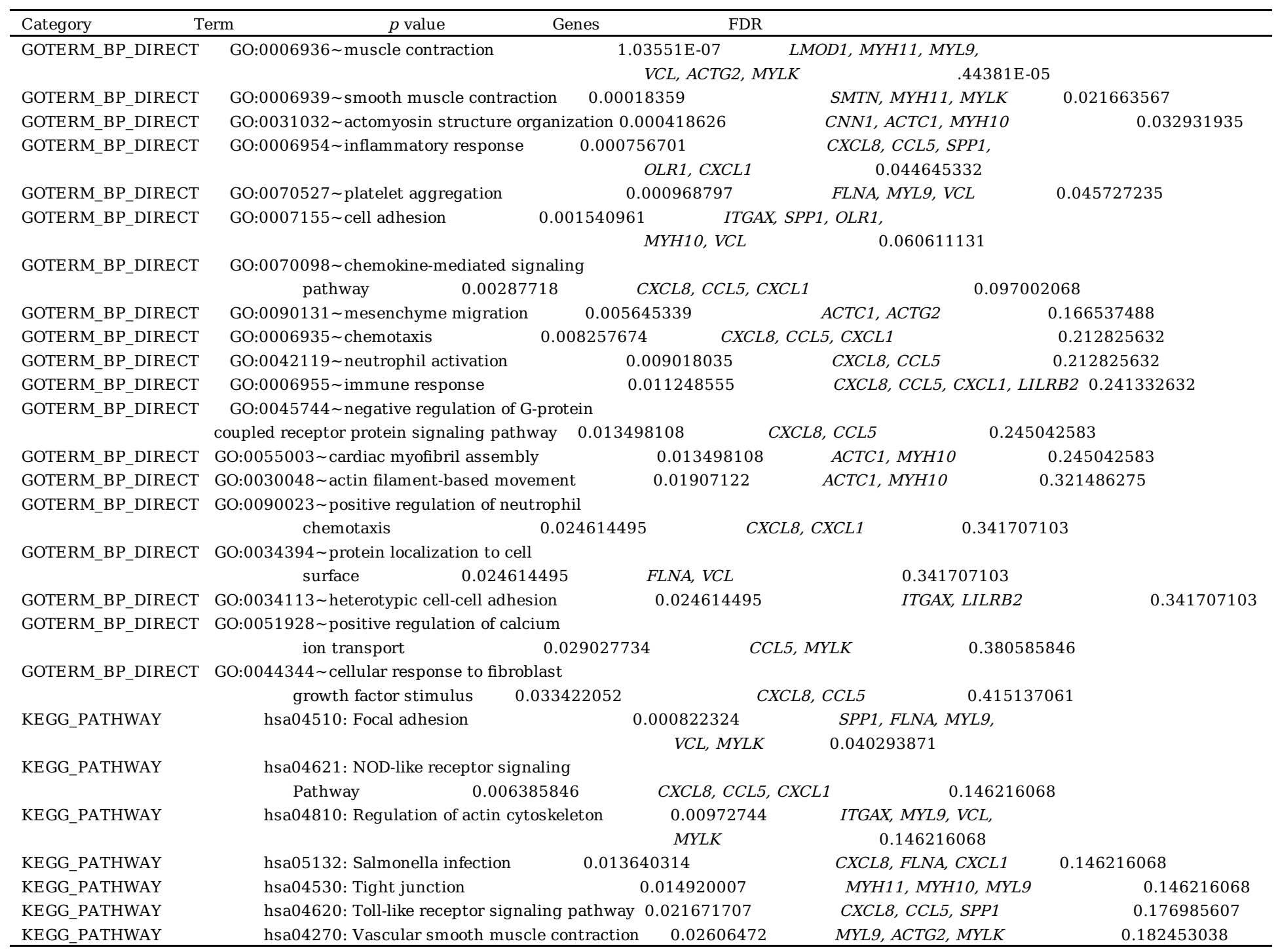

$\mathrm{p}$ value $\square 0.05$

\section{Figures}


A

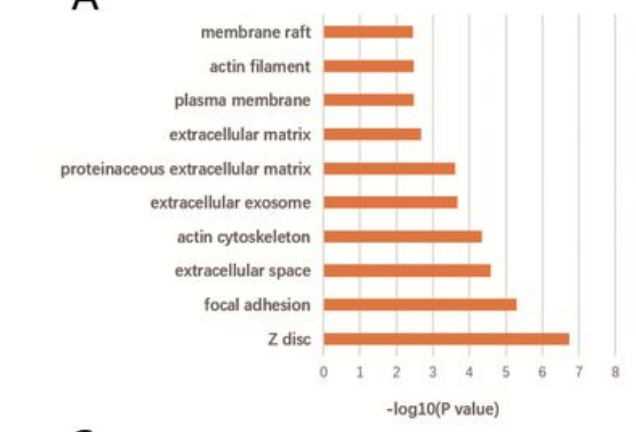

C

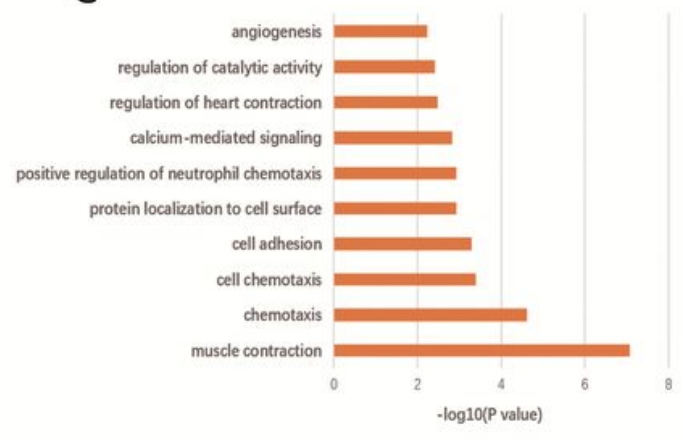

B

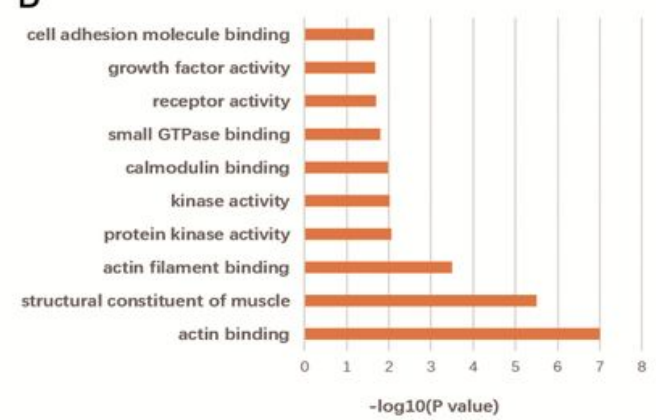

D

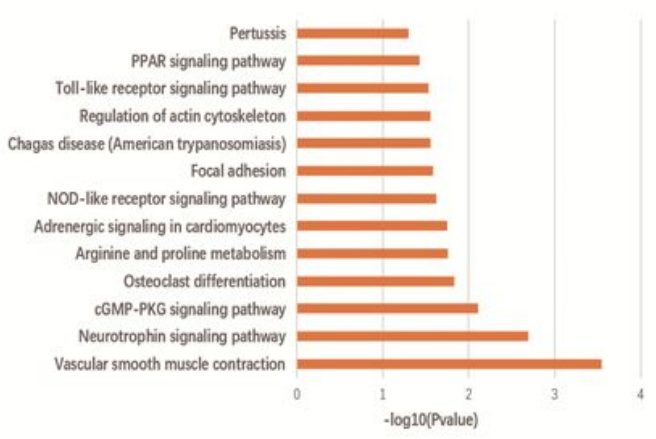

\section{Figure 1}

a Volcano Plot of GSE75436 b Volcano Plot of GSE7084 c Volcano Plot of GSE52093 d DEGs in Venny map 

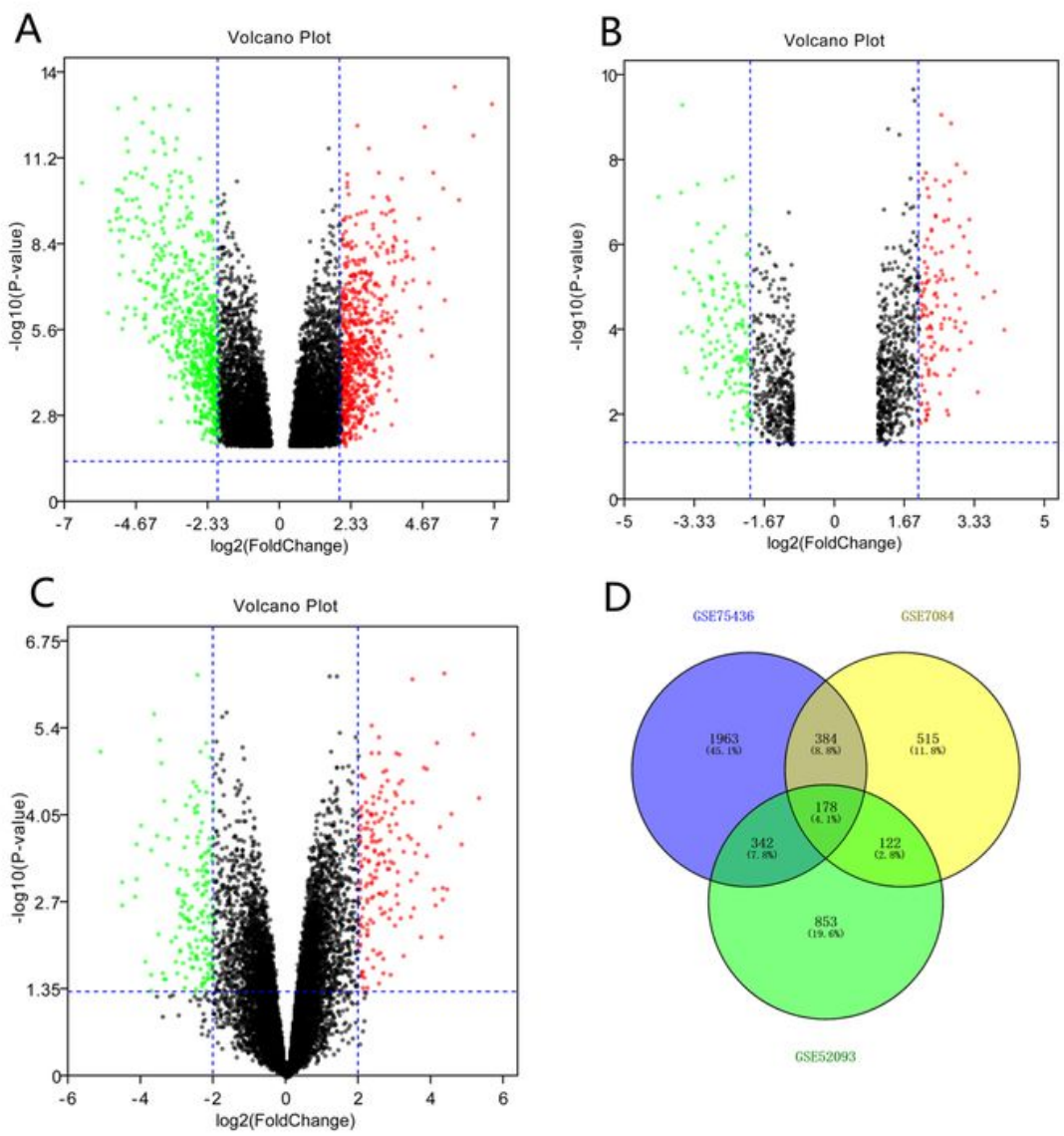

\section{Figure 2}

a cellular components(CC) b molecular functions(MF) c biology processes(BP) d KEGG pathway 


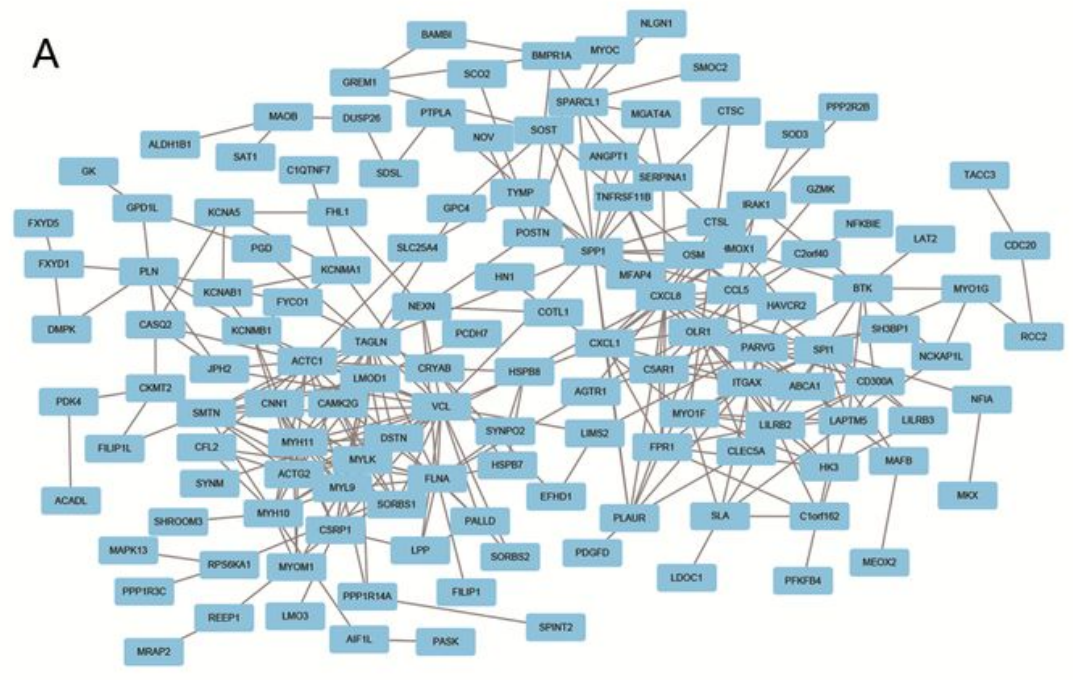

B

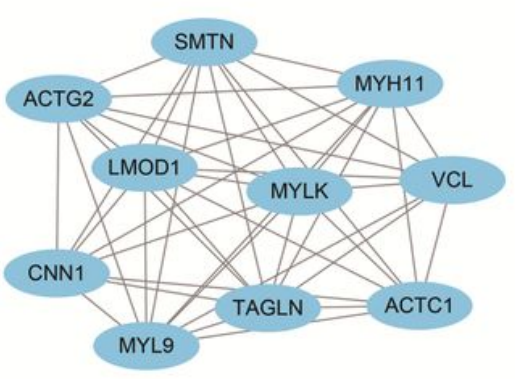

\section{Figure 3}

a PPI network of common DEGs b the most significant module 

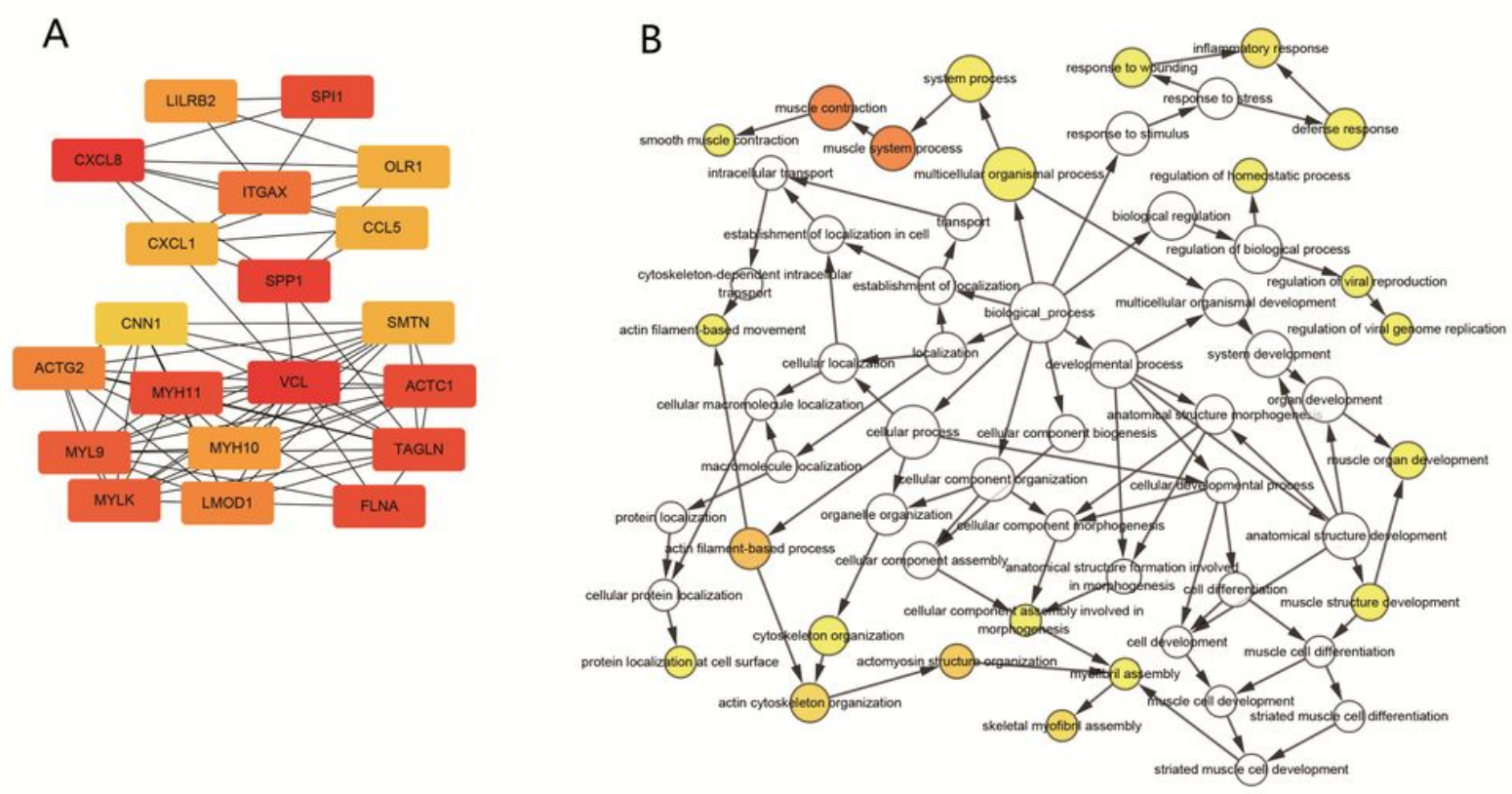

Figure 4

a PPI network of 20 hub genes b biological processes by BingGO analysis

\section{Supplementary Files}

This is a list of supplementary files associated with this preprint. Click to download.

- legend.docx 